\title{
Non-isothermal Activation Kinetics
}

\author{
Andrés Arango-Restrepo ${ }^{1,2}$, J. Miguel Rubi $^{1}$ \\ ${ }^{1}$ Secció de Física Estadística i Interdisciplinària, Departament de Física de la Matèria Condensada, \\ Facultat de Física, Universitat de Barcelona, Martí i Franquès 1 \\ 08028 Barcelona, Avd. Diagonal 647, 08028 Barcelona, Spain \\ E-mail: aarangor@unal.edu.co \\ ${ }^{2}$ Escuela de Química, Facultad de Ciencias, Universidad Nacional de Colombia \\ Calle 59A No 63-20, Bloque 21, Núcleo El Volador, Medellín, Colombia \\ E-mail:mrubi@ub.edu
}

Received: 30 March 2017; revised: 03 May 2017; accepted: 06 May 2017; published online: 30 September 2017

\begin{abstract}
We analyze the activation kinetics of a system immersed in a non-isothermal bath. Using mesoscopic nonequilibrium thermodynamics, we show that activation is not only driven by the affinity but also by the temperature gradient. Both thermodynamic forces play a role in the kinetics. The presence of a thermal gradient makes the detailed balance principle not fulfilled. We show that although the law of mass action holds locally, in terms of the local temperature, it is in general not valid globally, when the local values of the activation rate and the fugacity difference are replaced by their corresponding spatial averages. We analyze numerically the deviations of that global law from the actual activation kinetics as a function of the temperature gradient and the activation energy. Our analysis shows how to control the reaction rate by means of a temperature gradient.
\end{abstract}

Key words: kinetics, non-equilibrium thermodynamics, non-isothermal process, law of mass action

\section{INTRODUCTION}

Processes that need a minimum amount of energy to proceed, usually referred to as activated processes, take place in many cases under non-equilibrium conditions, under the influence of external driving forces. The presence of these forces modifies the free energy barrier thus leading to changes in the activation rates. Many examples can be found in physicochemical and biological systems. In these systems, mechanical forces may provide the energy that reactants need to transform into products $[1,2]$. They can also induce chemical changes in polymeric materials [3] and breaking and formation of chemical bonds [4]. The forces can also affect the kinetics of single molecules reactions [5]. An amount of tension applied at the ends of a RNA molecule breaks progressively the bonds, giving rise to new configurations of the molecule [6]. Those processes can be modeled by a particle crossing a free energy barrier that separates two welldifferentiated states located at the minima at each side of the barrier $[7,8,9]$ and are generically referred to as activated processes [8]. Those processes are nonlinear and cannot be treated by means of linear non-equilibrium thermodynamics [10].

Thermal activation may also take place under nonisothermal conditions, in the presence of a temperature gradient. An extension of Kramer's [7] model to the non-isothermal case was proposed in [11] and used in [12] to calculate the escape rate. The kinetics of nucleation processes is affected by the presence of gradients imposed to the metastable phase $[13,14]$ which results in changes of the nucleation rate. Thermal gradients may also affect the growth kinetics of lysozyme crystals/aggregates giving rise to a Soret-type effect [15].

In this article, we present a general formalism to treat activated processes taking place in a non-isothermal medium, 
in which both the affinity and the temperature gradient are the thermodynamic forces. We will show that the law of mass action (LMA) can be obtained from mesoscopic nonequilibrium thermodynamics $[16,17]$ which provides expressions for the activation rates in terms of the fugacity differences. The LMA is valid for sufficiently small values of the affinity, when the system reaches a quasi-stationary regime and can be considered as a two-level system. The nonisothermal kinetics will be analyzed by following a method similar to the one proposed for the isothermal case [18].

The article is organized as follows. In Section 2, we review the mesoscopic non-equilibrium thermodynamics approach to activated process showing how the LMA can be derived from the entropy production of the system. In Section 3 , we analyze the non-isothermal activated kinetics, whereas in Section 4 we present a numerical study on the effect that the thermal driving force exerts on the kinetics. Finally, in Section 5 we present our main conclusions.

\section{ACTIVATION KINETICS FROM MESOSCOPIC NON-EQUILIBRIUM THERMODYNAMICS}

Mesoscopic non-equilibrium thermodynamics [16, 17] provides a description of the activation kinetics. It quantifies the dissipation inherent to the process and gives a proof of the LMA. Unlike non-equilibrium thermodynamics [19], the theory assumes that at short time scales, the state of the system instead of jumping suddenly from the initial to the final state, it progressively transforms by passing through successive molecular configurations. These configurations can be parametrized by a reaction coordinate $\gamma$. At these time scales, one may assume that the reaction coordinate undergoes a diffusion process through a potential barrier separating the initial from the final states.

\section{1. The law of mass action from the entropy production}

At short time scales, an activated process, in which a substance or a state A transforms into another B, can be assimilated to a diffusion process along a reaction coordinate [16]. The corresponding entropy production is given by

$$
\sigma=-\frac{1}{T} J \frac{\partial \mu}{\partial \gamma}
$$

where $J$ is the diffusion current, $T$ is the temperature of the bath, assumed constant, and the chemical potential is

$$
\mu(\gamma, t)=k_{B} T \ln P(\gamma, t)+\varphi(\gamma)
$$

with $P(\gamma, t)$ the probability distribution, $k_{B}$ the Boltzmann constant and $\varphi(\gamma)$ the potential barrier. The flux obtained from this expression can be written in terms of the local fugacity defined along the reaction coordinate $z(\gamma) \equiv$ $\exp \left(\mu(\gamma) / k_{B} T\right)$ as

$$
J=-k_{B} L \frac{1}{z} \frac{\partial z}{\partial \gamma}
$$

with $L$ an Onsager coefficient. This expression can also be written as

$$
J=-D(z) \frac{\partial z}{\partial \gamma}
$$

where the diffusion coefficient is given by

$$
D=k_{B} \frac{L}{z}=k_{B} \frac{L}{P} e^{-\frac{\varphi}{k_{B} T}}
$$

We now assume that $D$ is constant and equal to $D_{0}$ and integrate from 1 (state A) to 2 (state B) to obtain the nonlinear kinetic law for the averaged flux [14]

$$
\begin{aligned}
\bar{J} \equiv \int_{1}^{2} J d \gamma & =-D_{0}\left(z_{2}-z_{1}\right)= \\
& =-D_{0}\left(\exp \left(\frac{\mu_{2}}{k_{B} T}\right)-\exp \left(\frac{\mu_{1}}{k_{B} T}\right)\right) .
\end{aligned}
$$

This equation can also be expressed as

$$
\bar{J}=J_{0}\left(1-e^{A / k_{B} T}\right),
$$

where $J_{0}=D_{0} \exp \left(\mu_{1} / k_{B} T\right)$ and $A=\mu_{1}-\mu_{2}$ is the affinity. Under quasi-stationary conditions, when the activation rate only depends on time, the actual value of the rate in (7) can be identified with its mean value. This equation thus corresponds to the LMA usually found in different activated processes [20, 21, 22].

\section{2. Detailed balance}

The scheme presented reproduces the results of the rate theory [9]. The equation (7) can be written in terms of the forward and backward reaction rates, $k_{+}$and $k_{-}$, as

$$
\bar{J}=k_{-} n_{2}-k_{+} n_{1}
$$

where $n_{1}$ and $n_{2}$ are the populations at the minima of the potential. The forward and backward reaction constants are given in terms of the potential:

$$
k_{+}=D_{0} \frac{e^{\varphi_{2} / k_{B} T}}{\overline{e^{\varphi / k_{B} T}}}
$$




$$
k_{-}=D_{0} \frac{e^{\varphi_{1} / k_{B} T}}{\overline{e^{\varphi / k_{B} T}}}
$$

Here the upper bars stands for average for all value of the reaction coordinate, as indicated in the first equality of (6).

The detailed balance condition follows from (8). By setting the overall rate equal to zero, one obtains $z_{1, e q}=z_{2, e q}$, or equivalently

$$
\frac{k_{+}}{k_{-}}=\frac{n_{2, e q}}{n_{1, e q}}
$$

where the $n$ 's are the equilibrium populations at both wells of the potential. This expression shows that the forward and backward reaction constants are not independent.

We can also analyze the case in which the transformation $A \rightleftarrows B$ is coupled to another process $C \rightleftarrows D$ in which the concentration of the components can be controlled. The current is in this case given by

$$
J=-D_{0}\left(z_{2}-z_{1}\right)=-D_{0}\left(z_{B} z_{D}-z_{A} z_{C}\right)
$$

where the initial and final fugacities are

$$
z_{1}=e^{\frac{\mu_{A}+\mu_{C}}{k_{B} T}}=z_{A} z_{C} \quad ; \quad z_{2}=e^{\frac{\mu_{B}+\mu_{D}}{k_{B} T}}=z_{B} z_{D}
$$

When both reactions are in equilibrium, the detailed balance condition imposes

$$
\frac{k_{+}}{k_{-}}=\frac{n_{B, e q} n_{D, e q}}{n_{A, e q} n_{C, e q}}
$$

Changes in the populations of states $\mathrm{C}$ and $\mathrm{D}$ drive the system away from equilibrium to a state in which detailed balance is not fulfilled.

The previous scheme can be generalized to the case of open and closed triangular reactions [23]. Fluctuations in the population densities at both wells can be analyzed by means of fluctuating hydrodynamics [18]. The coarse-graining of the description leads to violation of the fluctuation-dissipation theorem $[24,25]$.

\section{3. Kinetics at high affinities}

Kinetic theory shows that the evolution of a gas mixture is the result of the competition between two factors: the affinity, as the driving force, and the countless collisions between particles that tend to cancel the increase of momentum of the particles due to the force. The solution of the Boltzmann equation for a reacting gas through the Chapmann-Enskog expansion enables one to describe the transition towards nonequilibrium states [26]. The expansion establishes that the zeroth order term corresponds to the Maxwellian velocity distribution whereas the first order term is proportional to the affinity. It is found that for high values of the affinity, the LMA cease to be valid.

To obtain the reaction rates at higher values of the affinity, we notice that the diffusion coefficient in equation (5) may in general be a function of the chemical potential or equivalently on the fugacity: $D(z)$. Taking the average of the current as we did in (6), we arrive at

$$
\bar{J} \equiv \int_{1}^{2} J d \gamma=-D_{\text {eff }}\left(z_{2}-z_{1}\right)
$$

where the effective diffusion coefficient is given by

$$
D_{\text {eff }}=\frac{1}{\int_{1}^{2} D^{-1}(z) d \gamma}
$$

Eqs. (15) and (16) show how the LMA is affected by a non-constant diffusion coefficient in the space of the reaction coordinate.

The coefficient can also be written as

$$
D_{\text {eff }}\left(z_{1}, z_{2}\right)=D_{0}\left[1+f\left(z_{1}, z_{2}\right)\right]
$$

or equivalently as

$$
D_{\text {eff }}\left(\tilde{z}, z_{1}\right)=D_{0}\left[1+g\left(\tilde{z}, z_{1}\right)\right]
$$

where $\tilde{z}=z_{2}-z_{1}$. In the particular case of a diffusion coefficient linear in $z$ one obtains

$$
J(t)=-D_{0}\left(z_{2}-z_{1}\right)-D_{1} \frac{1}{2}\left(z_{2}^{2}-z_{1}^{2}\right)
$$

The effective diffusion coefficient is then given by

$$
D_{\text {eff }}=D_{0}\left\{1+\frac{D_{1}}{D_{0}}\left(z_{1}+\frac{1}{2} \tilde{z}\right)\right\}
$$

As in the rate theory, the current also can be expressed in terms of forward and backward currents in the form

$$
\bar{J}(t)=\tilde{k}_{+} n_{2}-\tilde{k}_{-} n_{1}
$$

where the forward and backward reaction rates are given by

$$
\begin{aligned}
\tilde{k}_{+} & =D_{0}\left\{1+\frac{D_{1}}{\frac{e^{\varphi / k_{B} T}}{D_{0}}}\left(2 z_{1}+\frac{1}{2}\left(z_{2}-z_{1}\right)\right)\right\} e^{\varphi_{2} / k_{B} T} \\
& =D_{\text {eff }} e^{\varphi_{2} / k_{B} T} \\
\tilde{k}_{-} & =D_{0}\left\{1+\frac{D_{1}}{\overline{e^{\varphi / k_{B} T}} D_{0}}\left(2 z_{1}+\frac{1}{2}\left(z_{2}-z_{1}\right)\right)\right\} e^{\varphi_{1} / k_{B} T} \\
& =D_{\text {eff }} e^{\varphi_{1} / k_{B} T}
\end{aligned}
$$

We can then see that at higher values of the chemical driving force, the reaction rates depend on the affinity through the driving force $\left(z_{2}-z_{1}\right)=-e^{\mu_{1} / k_{B} T}\left(1-e^{A / k_{B} T}\right)$, 


$$
D_{\text {eff }}=D_{0}\left\{1+\frac{D_{1} e^{\mu_{1} / k_{B} T}}{\overline{e^{\varphi / k T}} D_{0}}\left(2-\frac{1}{2}\left(1-e^{A / k_{B} T}\right)\right)\right\}
$$

and therefore, the law of mass action ceases to be valid. Therefore, the detailed balance principle is not fulfilled. The previous equations generalize the rate theory to the case of high affinities [27].

Our analysis based on the entropy production in the reaction coordinate space (1) is consistent with the kinetic theory of gases which shows that up to first order in the Chapmann-Enskog expansion the entropy production can still be expressed in terms of flux-force pairs [26]. This property is not guaranteed for higher values of the driving force when one has to consider higher order terms in the expansion.

\section{NON-ISOTHERMAL ACTIVATION}

The presence of thermal inhomogeneities in the bath affects the kinetics of an activated process adding a new driving force, the temperature gradient. In the framework of mesoscopic non-equilibrium thermodynamics, the description must in general be made in terms of a probability distribution that depends not only on the reaction coordinate but on the positions and the velocities of the particles: $P(\underline{\Gamma}, t)$, where $\underline{\Gamma}(\gamma, \vec{r}, \vec{u})$ is a phase space vector. The dissipation in the system contains the contribution due to the activated process, obtained in the previous section, and those corresponding to heat conduction and diffusion.

For nucleation or self-assembly processes in which the reaction coordinate is simply the number of atoms in a cluster or the number of building blocks in the structure, $\gamma \equiv n$, the entropy production is given by [13]

$$
\begin{aligned}
\sigma(\vec{r}, t) & =-\frac{1}{T^{2}} \vec{J}_{q}^{\prime} \cdot \nabla T-\frac{1}{T} \int \vec{J}_{u} \cdot \nabla_{u} \mu d n d \vec{u} \\
& -\frac{1}{T} \int J_{n} \nabla_{n} \mu d n d \vec{u}
\end{aligned}
$$

where the chemical potential is now

$$
\mu(\underline{\Gamma}, t)=k_{B} T(\vec{r}) \ln n(\underline{\Gamma}, t)+\varphi(\gamma)+\frac{1}{2} m u^{2}
$$

and the modified heat flux contains the contribution of the energy of the particles and is given by

$$
\vec{J}_{q}=\vec{J}_{q}-\int f \vec{u}\left[\Delta H(n)+\frac{1}{2} m(n) u^{2}\right] d n d \vec{u}
$$

Here $\Delta H(n)$ is the enthalpy of a cluster formed by $n$ particles. The temperature of the bath may now depend on position $\vec{r}$.
The fluxes involved in the entropy production given in Eq. (24) are of different tensorial nature: a scalar for the activation rate and a vector for the heat flux. Under isotropic conditions both currents are not coupled. An exception is the case of active transport in the $\mathrm{Ca}^{2+}-A T P$ ase under a temperature difference in which ions move along a $1 D$ channel and the rate is a scalar [20]. Due to this decoupling, the activation rate can be obtained from the contribution of the activation process to the entropy production

$$
\sigma_{a c t}(\underline{\Gamma}, t)=-\frac{1}{T(\vec{r})} J \frac{\partial \mu}{\partial \gamma}
$$

In the non-isothermal case, we can define a fugacity as: $z(\underline{\Gamma}, t) \equiv \exp \left(\mu(\underline{\Gamma}, t) / k_{B} T(\vec{r})\right)$, where the phase-space vector is now $\underline{\Gamma}(\gamma, \vec{r})$ and $T(\vec{r})$ is the stationary temperature. In terms of this quantity, the expression for the activation rate is

$$
J(\underline{\Gamma}, t)=-D \frac{\partial z(\underline{\Gamma}, t)}{\partial \gamma}
$$

Without assuming that $D$ is a constant due to the fact that the temperature gradient modifies the potential barrier, and writing $D=D_{0} e^{-\varphi(\gamma) / k_{B} T(\vec{r})}$, we express the activation rate as

$$
J(\underline{\Gamma}, t)=-D_{0} e^{\frac{-\varphi(\gamma)}{k_{B} T(\vec{r})}} \frac{\partial z(\underline{\Gamma}, t)}{\partial \gamma}
$$

Integrating now equation (29), as we did in the two previous sections, assuming quasi-stationary state in the space of the reaction coordinate and a constant diffusion coefficient, we obtain

$$
\bar{J}(\vec{r}, t)=-\frac{D_{0}}{\int_{0}^{1} e^{\frac{-\varphi(\gamma)}{k_{B} T(\vec{r})}} d \gamma}\left(z_{2}(\vec{r}, t)-z_{1}(\vec{r}, t)\right),
$$

or equivalently

$$
\begin{aligned}
\bar{J}(\vec{r}, t)= & \frac{D_{0}}{\int_{0}^{1} e^{\frac{-\varphi(\gamma)}{k_{B} T(\vec{r})}} d \gamma} \\
& \times\left(e^{\frac{-\mu_{2}(\vec{r}, t)}{k_{B} T(\vec{r})}}-e^{\frac{-\mu_{1}(\vec{r}, t)}{k_{B} T(\vec{r})}}\right)
\end{aligned}
$$

This expression constitutes a local formulation of the LMA. A more general expression of the LMA in reactiondiffusion systems in the presence of temperature and concentration gradients was given in [28].

The activation rate can also be written as

$$
\bar{J}=\hat{k}_{-} P_{2}-\hat{k}_{+} P_{1}
$$


Tab. 1. Parameters used in the simulations

\begin{tabular}{l|l}
\hline Parameter (dimensionless) & Value \\
\hline Dimensionless Standard Affinity: $\frac{\mu^{0}{ }_{2}-\mu^{0}{ }_{1}}{k_{B} T}$ & 2 \\
\hline Initial values of the probabilities $P_{1}(0)=P_{2}(0)$ & 0.5 \\
\hline Activation Energy: $\frac{E_{a}}{k_{B} T}$ & $1-9$ \\
\hline Temperature Dimensionless Gradient: $\frac{d \hat{T}}{d \hat{x}}=\frac{\nabla T}{T_{0}}$ & $0-2$ \\
\hline
\end{tabular}

$$
\frac{\hat{k}_{+}}{\hat{k}_{-}}=e^{\frac{-\Delta \varphi}{k_{B} T(\vec{r}}}
$$

where $\Delta \varphi=\varphi(1)-\varphi(2)$. When the latent heat involved in the activation process is negligible, the temperature follows from the Fourier heat equation, whose stationary solution is given by

$$
T(\vec{r})=T_{0}+\vec{r} \cdot \vec{\nabla} T
$$

Here $T_{0}$ is a reference temperature. Using this expression, equation (34) can be rewritten in terms of the temperature gradient as

$$
\frac{\hat{k}_{+}}{\hat{k}_{-}}=e^{\frac{-\Delta \varphi}{k_{B} T_{0}}}\left(\frac{1}{1+\vec{r} \cdot \vec{\nabla} T / T_{0}}\right)
$$

Defining the effective potential

$$
\Delta \varphi_{\mathrm{eff}}=\frac{\Delta \varphi}{\left(1+\vec{r} \cdot \vec{\nabla} T / T_{0}\right)}
$$

We write (35) in terms of the effective potential

$$
\frac{\hat{k}_{+}}{\hat{k}_{-}}=e^{\frac{\Delta \varphi_{\mathrm{eff}}}{k_{B} T_{0}}}
$$

Up to linear order in $\varepsilon \equiv \vec{r} \cdot \vec{\nabla} T / T_{0}$, one obtains

$$
\frac{\hat{k}_{+}}{\hat{k}_{-}}=\left(\frac{\hat{k}_{+}}{\hat{k}_{-}}\right)_{\nabla T=0} e^{-\frac{\Delta \varphi}{k T_{0}} \varepsilon}
$$

This expression shows the correction of the temperature gradient to the detailed balance principle. Finally, using the effective potential, the current is written as

$$
\bar{J}(\vec{r}, t)=-\frac{D_{0}}{\int_{0}^{1} e^{\frac{\Delta \varphi_{\mathrm{eff}}}{k_{B} T_{0}}} d \gamma} \Delta z(\vec{r}, t)
$$

\section{NUMERICAL ANALYSIS OF NON-ISOTHERMAL ACTIVATION}

In this Section, we will analyze numerically the effect that the presence of a temperature gradient exerts on the activation kinetics. Spatial diffusion along the direction of the temperature gradient and reaction heat are assumed to be small enough as to be considered not determinant in the process. Diluted and reactive systems in narrow channels with temperature gradient [29], fluids in micro-channels, fluids in the presence of linear temperature gradient [30], and cyclic reactions in micro-channels with radial temperature gradients [31] are some examples which fulfill the two assumptions.

We will restrict our analysis to the $1 D$ case and will solve the continuity equation

$$
\frac{\partial}{\partial t} P(\gamma, x, t)=-\frac{\partial}{\partial \gamma} J(\gamma, x, t)
$$

to obtain the probability density $P(\gamma, x, t)$ which will be obtained in the quasi-stationary case, when the potential barrier is sufficiently high. Integrating equation (40) from $\gamma=\gamma^{*}$ to $\gamma=1$, where $\gamma^{*}$ denotes the value of the coordinate at the transition state, the resulting evolution of the probability at state 2 is

$$
\frac{d}{d t} P_{2}(x, t)=-\bar{J}(x, t)
$$

and due to probability conservation, that at state 1 is given by

$$
\frac{d}{d t} P_{1}(x, t)=\bar{J}(x, t)
$$

We will now analyze the deviation of the law of mass action in the presence of a temperature gradient in one dimension, where the temperature is fixed at both boundaries, yielding the linear stationary temperature profile (35). In $1 D$, 
Tab. 2. $\chi^{*}$ as a function of the fugacities difference, temperature gradient and activation energy

\begin{tabular}{l|l|l|l|l}
\hline$\langle\Delta z\rangle$ & $\nabla T / T_{0}$ & $E_{a / k_{B} T}$ & $\chi^{*}$ & Validity of LMA \\
\hline$\langle\Delta z\rangle \rightarrow 0$ & $\forall \nabla T / T_{0}$ & $\forall E_{a} / k_{B} T$ & $\chi^{*} \rightarrow 1$ & valid \\
\hline \multirow{3}{*}{$\forall\langle\Delta z\rangle>0$} & $\nabla T / T_{0} \rightarrow 0$ & $\forall E_{a} / k_{B} T$ & $\chi^{*} \rightarrow 1$ & valid \\
\cline { 2 - 5 } & $0<\nabla T / T_{0}<0.5$ & $\forall E_{a} / k_{B} T$ & $\chi^{*}-1 \propto E_{a}^{2} \nabla T$ & Not valid \\
\cline { 2 - 5 } & $\nabla T / T_{0} \geq 0.5$ & $E_{a} / k_{B} T<3$ & $\chi^{*}-1 \propto E_{a}^{2} \nabla T$ & Not valid \\
\cline { 2 - 5 } & $\nabla T / T_{0} \geq 0.5$ & $E_{a} / k_{B} T \geq 3$ & $\chi^{*}-1 \propto E_{a}^{2} \nabla T^{1 / 2}$ & Not valid \\
\hline
\end{tabular}

the quasi-stationary current in (40) can be obtained in a similar way as we did in Section 2:

$$
\bar{J}(x, t)=-\frac{D_{0}}{\int_{0}^{1} e^{\frac{\Delta \varphi_{\text {eff }}}{k_{B} T_{0}}} d \gamma} \Delta z(x, t)
$$

with $\Delta z=z_{2}-z_{1}$. The current will be expressed in terms of the dimensionless time $\tau=t D_{0}$, the dimensionless position $\hat{x}=x / L$, where $L$ is the size of the system, and the dimensionless temperature $\hat{T}=T / T_{0}$. The values of the parameters used in the numerical analysis are shown in Table1.

The dimensionless current is defined as

$$
\bar{J}(\hat{x}, \tau)=-\hat{D}(\hat{x}) \Delta z(\hat{x}, \tau)
$$

where $\hat{D}(\hat{x})=\left[\int_{0}^{1} e^{\frac{\Delta \varphi_{\text {eff }}}{k_{B} T_{0}}} d \gamma\right]^{-1}$ and $\Delta \varphi_{\text {eff }}$ depends on the activation energy $E_{a}$. To compute $\varphi(\gamma)$, we will use a bistable potential barrier. The space average of the current is given by

$$
\langle\bar{J}\rangle=-\langle\hat{D} \Delta z\rangle
$$

As $\hat{D}(\hat{x})>0$, and $\Delta z \leq 0$, one has

$$
\langle\bar{J}\rangle \leq \stackrel{J}{J}^{*}=-\langle\hat{D}\rangle\langle\Delta z\rangle
$$

with $\langle\bar{J}\rangle=J^{*}$, when $\hat{D}(\hat{x})$ or $\Delta z(\hat{x}, \tau)$ are constant in position. This happens for small values of the driving forces: $\nabla T / T_{0} \rightarrow 0$, and $\Delta z \rightarrow 0$. In Fig. 1, we show the behavior of the current $\langle\bar{J}\rangle$ against $\langle\Delta z\rangle$.

As follows from Fig. 1, by increasing the temperature gradient leads to the failure of the linear relationship between current and fugacity differences necessary for the LMA to be valid. The law represents a good approximation at small values of the gradient. For instance, for $E_{a}=3 k_{B} T$, if the gradient is less than $9.1 \mathrm{~K} / \mathrm{m}$, the error is less than $0.1 \%$ for all values of $\langle\Delta z\rangle$. But for $E_{a}=3 k_{B} T$ and temperature gradient higher than $149 \mathrm{~K} / \mathrm{m}$, the error is greater than $10 \%$, for all $\langle\Delta z\rangle$. Even the error is more than $50 \%$ if the gradient is higher than $498 \mathrm{~K} / \mathrm{m}$.

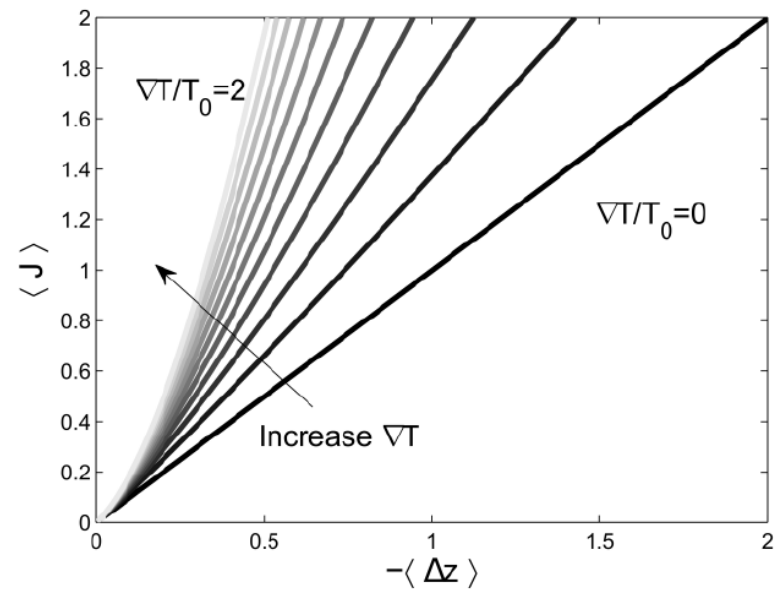

Fig. 1. Average current vs average fugacity difference for $\nabla T / T / 0$ from 0 to 2, increasing each 0.2 . Grey lines are lighter as the temperature gradient increases

Fig. 1 shows that the averaged current follows the power law

$$
\langle\bar{J}\rangle=-D_{\text {eff }}\langle\Delta z\rangle^{\chi}
$$

where $D_{\text {eff }} \equiv\langle\hat{D}\rangle$. The exponent $\chi$ measures deviations from the global LMA behavior which corresponds to $\chi=1$. To quantify the violation of LMA when a temperature gradient is applied, we represent in Fig. 2 the deviation factor $\chi$ against $\langle\Delta z\rangle$, for different temperature gradients. We verify that at low values of $\langle\Delta z\rangle, \chi$ tends to 1 . A maximum value of $\chi$ is observed at large enough values of $\nabla T$. The maxima displace to the left as the temperature gradient increases. This means that even for small $\langle\Delta z\rangle$, LMA ceases to be valid for high temperature gradients. 
For a given temperature gradient, the maximum value of $\chi, \chi^{*}$, indicates maximum deviation of the LMA. In Fig. 3, we show the behavior of $\chi^{*}$ as a function of the temperature gradient $\nabla T$, for different activation energies $E_{a}$.

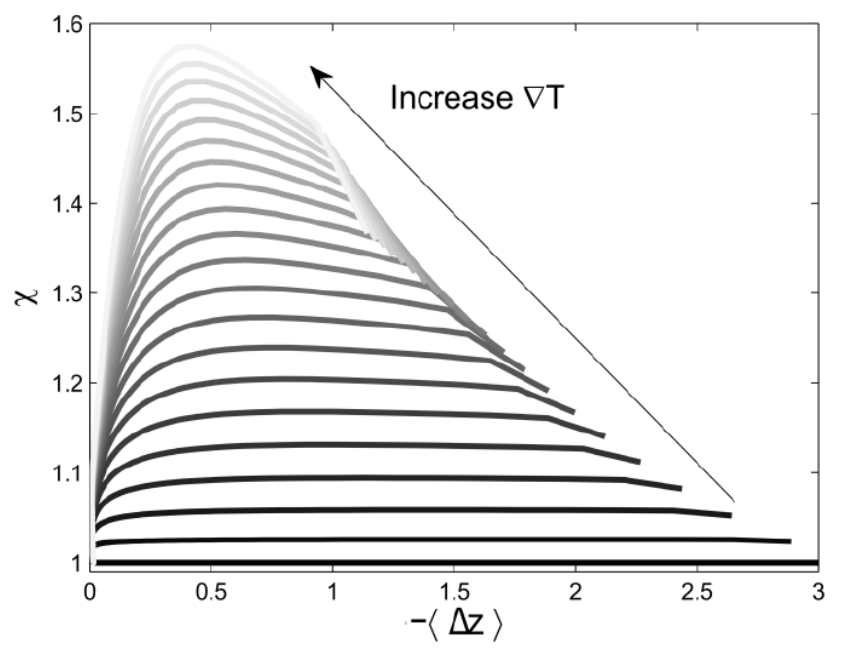

Fig. 2. The power law exponent $\chi$ versus $\langle\Delta z\rangle$ for different values of $\nabla T$

At low values of the gradient, the location of the maxima is a linear function of it. This behavior results from the fact that at low temperature gradients changes in the diffusivity vary linearly with the gradient. For $\nabla T>0.5 T_{0}$, the behavior is nonlinear in $\nabla T$. Moreover, $\chi^{*}$ increases when $E_{a}$ increases, due to the fact that for high activation energies, $D_{\text {eff }}$ is more sensitive to the temperature gradient. In Fig. 4, we show $\chi^{*}$ as a function of the temperature gradient and the activation energy.

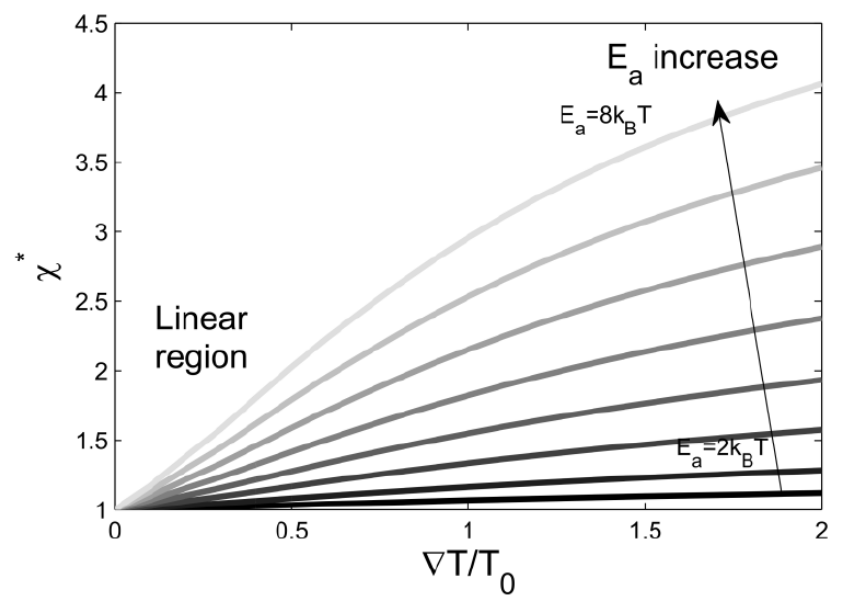

Fig. 3. $\chi^{*}$ versus $\nabla T$ for different activation energies $E_{a}$

In Table 2, where summarize the behavior of $\chi^{*}$ as a function of the fugacity differences, temperature gradient and activation energy which enables us to infer the conditions under which LMA is valid. We found that the deviation of the LMA is quadratic in the energy activation. Further, for low activation energy, the deviation of LMA is more sensitive to an increase of the temperature gradient than to an increase of the activation energies.

From the values of $\chi^{*}$, we can compute the error committed in assuming that the LMA is valid in a non-isothermal system. In Fig. 5, we show the dependence of the error percentage, defined as \%error $=\left(\chi^{*}-1\right) 100 \%$, on the activation energy and the temperature gradient. We see that for high activation energies, in order to keep a small error, the corresponding gradient must decrease. This is so because for high energies, $D_{\text {eff }}$ increases faster than for low energies.

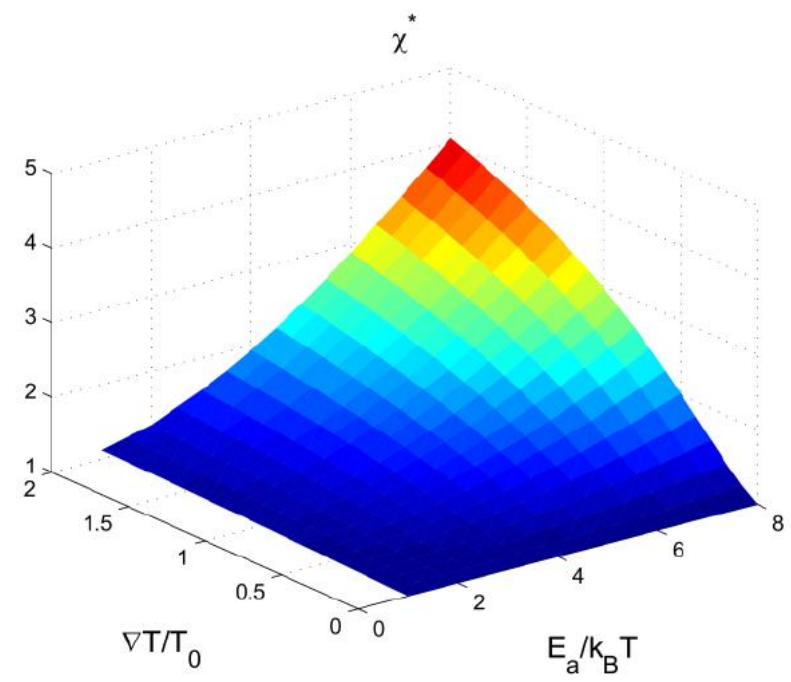

Fig. 4. Representation of the surface: $\chi^{*}\left(\nabla T, E_{a}\right)$

\section{CONCLUSIONS}

In this article, we have analyzed activated processes taking place under non-isothermal conditions, when nonequilibrium states originate not only from chemical potential differences but also from the presence of a thermal gradient. We have shown that the symmetry breaking inherent to the presence of the thermal gradient leads to violation of the detailed balance principle. This implies that fixing say the forward reaction rate the backward one can be controlled by changing the temperature gradient.

We have analyzed the effect that a temperature gradient exerts on the activation rate and computed the difference between the spatial average of the rate and the value predicted by the LMA obtained under isothermal conditions. We have found that when the activation energy of the processes increases, deviations from LMA become more important. Such deviations are significant for temperature gradients higher than $1 \mathrm{~K} / \mathrm{cm}$.

The formalism presented provides a general scheme to analyze far-from-equilibrium activated processes in the presence of thermal inhomogeneities. It offers applications to a 


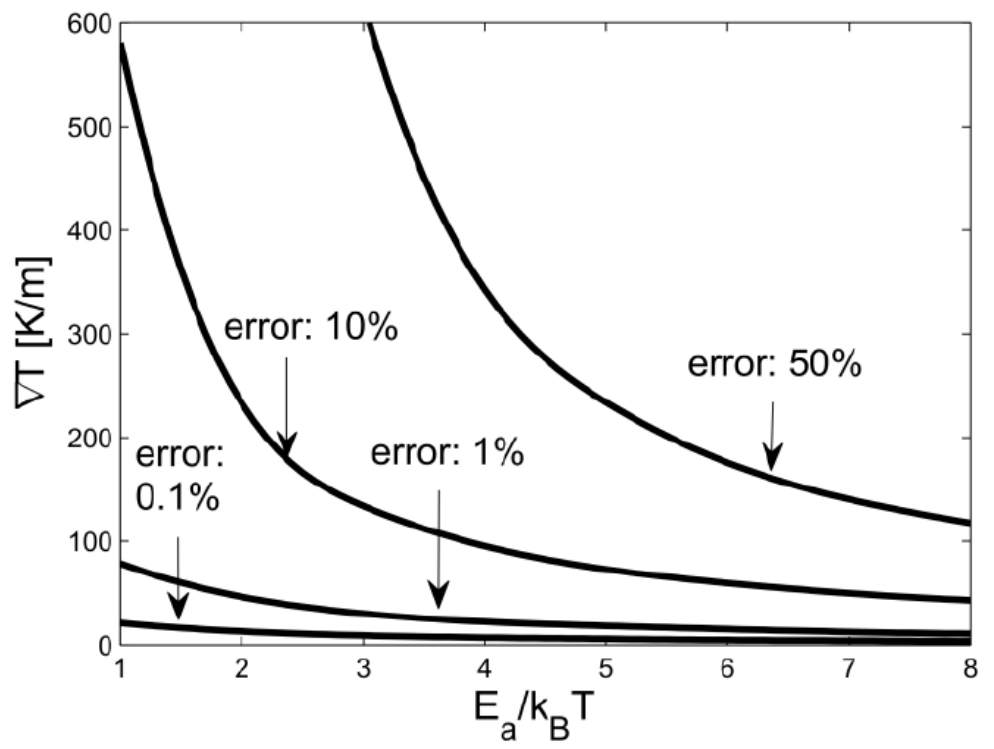

Fig. 5. Error percentage for different values of $\nabla T$ and $E_{a}$

wide variety of situations including chemical and biochemical reactions, nucleation and self-assembly processes. Our analysis may also contribute to the study of non-isothermal processes taking place in complex systems such as a living cell. Due to cellular processes such as cell division, gene expression, enzyme reactions and metabolism, the temperature of living cells is not homogeneous and exhibits a dynamical behavior [32]. Another interesting application of the theory presented concerns the analysis of nano-thermometers [33] used in living cells [34] and meso-systems [35,36] to measure the temperature. From the proposed kinetic expression, it could be possible to calibrate a nano-thermometer for nonequilibrium conditions. Moreover, the kinetic expressions obtained could be helpful to study the thermal-dynamics of pathological cells $[32,34]$, which are warmer and kinetically different from healthy cells.

\section{Bibliography}

[1] C. R. Hickenboth, J.S. Moore, S.R. White, N.R. Sottos, J. Baudry, and S.R. Wilson, Biasing reaction pathways with mechanical force, Nature, 446 , 423-427 (2007).

[2] B.M. Rosen, V. Percec, A reaction to stress, Nature, 446, 381-382 (2007).

[3] M.M. Caruso, D.A. Davis, Q. Shen, S.A. Odom, N.R. Sottos, S.R. White and J.S. Moore, Mechanically-Induced Chemical Changes in Polymeric Materials, Chem. Rev., 109, 57555798 (2009).

[4] E.M. Lupton, C. Bräuchle, and I. Frank, Understanding Mechanically Induced Chemical Reactions, NIC Symposium 2006 , edited by G. Münster, D. Wolf and M. Kremer, NIC Series, 32, 57-64 (2006).

[5] I. Tinoco and C. Bustamante, The effect of force on thermodynamics and kinetics of single molecule reactions, Biophys. Chem., 101-102, 513-533 (2002).
[6] J.M. Rubi, D. Bedeaux, and S. Kjelstrup, Unifying Thermodynamic and Kinetic Descriptions of Single-Molecule Processes: RNA Unfolding under Tension, J. Phys. Chem B, 111, 9598-9602 (2007).

[7] H. Kramers., Brownian motion in a field of force and the diffusion model of chemical reactions, Physica, 7, 284-304 (1940).

[8] S. Glasstone, K. J. Laidler and H. Eyring, The Theory of Rate Processes: The Kinetics of Chemical Reactions, Viscosity, Diffusion and Electrochemical Phenomena (McGraw-Hill, New York, 1941).

[9] P. Hänggi, P. Talkner, and M. Borkevec, Reaction-rate theory: fifty years after Kramers, Rev. Mod. Phys., 62, 251-341 (1990).

[10] T.L. Hill, Free Energy Transduction and Biochemical Cycle Kinetics (Dover, New York, 1989).

[11] H. Dekker, Nonequilibrium thermodynamics of nonisothermal activation: escape over mesoscopic barriers. I. The stochastic process, Physica A, 173, 381-410 (1991).

[12] H. Dekker, Nonequilibrium thermodynamics of nonisothermal activation: escape over mesoscopic barriers. II. The escape rate, Physica A, 173, 411-444 (1991).

[13] D. Reguera and J.M. Rubi, Homogeneous nucleation in inhomogeneous media. I. Nucleation in a temperature gradient, J. Chem. Phys, 119, 9877-9887 (2003.

[14] D. Reguera and J.M. Rubi, Homogeneous nucleation in inhomogeneous media. II. Nucleation in a shear flow, J. Chem. Phys. 119, 9888-9893 (2003).

[15] I. Santamaria-Holek, A. Gadomski and J.M. Rubi, Controlling protein crystal growth rate by means of temperature, $\mathrm{J}$. Phys. Cond. Matt., 23, 235101 (2011).

[16] D. Reguera, J.M. Rubi and J.M.G. Vilar, The Mesoscopic Dynamics of Thermodynamic Systems, J. Phys. Chem. B, 109, 21502-21515 (2005).

[17] Vilar, J. M. G., and Rubi, J. M., Proc. Nat. Acad. Sci., Thermodynamics "beyond" local equilibrium, 98, 11081-11084 (2001).

[18] I. Pagonabarraga, A. Perez-Madrid and J.M. Rubi, Fluctuating hydrodynamics approach to chemical reactions, Physica A, 237, 205-219 (1997). 
[19] S.R. de Groot and P. Mazur, Non-Equilibrium Thermodynamics (Dover, New York, 1984).

[20] S. Kjelstrup, J.M. Rubi, D. Bedeaux, Energy dissipation in slipping biological pumps, Phys. Chem. Chem. Phys., 7, 4009-4018 (2005).

[21] G. Gomila and J.M. Rubi, Relation for the nonequilibrium population of the interface states: Effects on the bias dependence of the ideality factor, J. Appl. Phys., 81, 2674-2681 (1997).

[22] J.M. Rubi and S. Kjelstrup, Mesoscopic nonequilibrium thermodynamics gives the same thermodynamic basis to ButlerVolmer and Nernst equations. J. Phys. Chem. B, 107, 1347113477 (2003)

[23] J.M. Rubi, D. Bedeaux, S. Kjelstrup, and I. Pagonabarraga, Chemical Cycle Kinetics: Removing the Limitation of Linearity of a Non-equilibrium Thermodynamic Description, Int. J. Thermophys., 34, 1214-1228 (2013).

[24] A. Perez-Madrid, D. Reguera, and J.M. Rubi, Origin of the violation of the fluctuation-dissipation theorem in systems with activated dynamics, Physica A, 329, 357-364 (2003).

[25] J.W. Dufty and J.M. Rubi, Generalized Onsager symmetry, Phys. Rev. A, 36, 222-225 (1987).

[26] J. Ross, and P. Mazur, Some Deductions from a Formal Statistical Mechanical Theory of Chemical Kinetics, J. Chem. Phys., 35, 19-28 (1961).

[27] J.M. Rubi and A. Perez-Madrid, Far-from equilibrium kinetic processes, J. Non-Equilib. Thermodyn., 40, 275-281 (2015).

[28] D. Bedeaux, I. Pagonabarraga, J.M. Ortiz de Zárate, J.V. Sengers, and S.Kjelstrup, Mesoscopic non-equilibrium thermo- dynamics of non-isothermal reaction-diffusion, Phys. Chem. Chem. Phys., 12, 12780-12793 (2010).

[29] K. Maruta, T. Kataoka, N.I. Kim, S. Minae, and R. Fursenko, Characteristics of combustion in a narrow channel with a temperature gradient, Proc. Combust. Inst., 30 2429-2436 (2005).

[30] Y. Mao, T. Ynag and P.S. Cremer, A microfluidic device with a linear temperature gradient for parallel and combinatorial measurement, J. Am. Chem. Soc., 124, 4432-4435 (2002).

[31] J.Y. Cheng, C.J. Hsieh, Y.C. Chuang and J.R. Hsieh, Performing microchannel temperature cycling reactions using reciprocating reagent shuttling along a radial temperature gradient, Analyst., 130, 931-940 (2005).

[32] C. Gota, K. Okabe, T. Funatsu, Y. Harada, S. Uchiyama, Hydrophilic Fluorescent Nanogel Thermometer for Intracellular Thermometry, J. Am. Chem. Soc. 131, 2766-2767 (2009).

[33] S. H, Kim, et al. Micro-Raman thermometry for measuring the temperature distribution inside the microchannel of a polymerase chain reaction chip, J. Micromech. Microeng., 16, 526-530 (2006).

[34] P. Low, B.Kim, N. Takama, \& C.Bergaud, High-SpatialResolution Surface-Temperature Mapping Using Fluorescent Thermometry, Small 4, 908-914 (2008).

[35] F. Vetrone, et al., Temperature Sensing Using Fluorescent Nanothermometers, ACS Nano 6, 3254-3258 (2010).

[36] S. Wang, S. Westcott, \& W. Chen, Nanoparticle Luminescence Thermometry, J. Phys. Chem. B 106, 11203-11209 (2002).

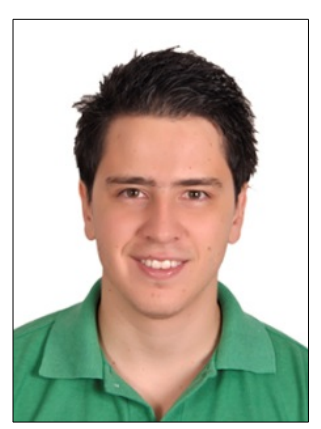

Andrés Arango-Restrepo graduated with the highest distinction in Chemical Engineering from the National University of Colombia in 2015; his undergraduate thesis was awarded as one of the best for the National University of Colombia in 2015. He obtained the highest distinction ("Laureate Thesis") in the Master degree of Science-Chemistry from the National University of Colombia in 2017 by his work on Non-Equilibrium Self-Assembly Processes to describe the thermodynamics of structures formation at the mesoscale.

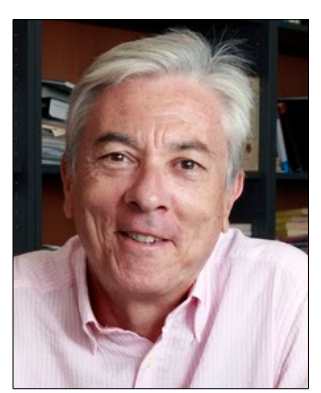

J. Miguel Rubi is Professor of Condensed Matter Physics in the University of Barcelona. In 2003, the Alexander von Humboldt Foundation awarded him the von Humboldt Prize, and in 2003 the University of Trondheim awarded him the Onsager Medal. He was appointed CAS Fellow 2007/2008 by the Centre for Advanced Study at the Norwegian Academy of Sciences and Letters. In 2010, the Institució Catalana de Recerca i Estudis awarded him an ICREA Prize. He has been appointed Onsager Professor at the University of Trondheim, Sandoval Vallarta Professor at UAM, Marcos Moshinsky Professor at UNAM and Institute of Physics Professor at San Luis Potosí of Mexico. In 2012 and 2013 he was Visiting Professor at CETHIL-INSA, Lyon, and in 2014 he was Visiting Professor at L'École Polytechnique, Paris. Professor Rubi was appointed Leverhulme Professor at Imperial College, London in 2013-14. In 2016, the Norwegian University of Science and Technology NTNU awarded him the degree of Doctor Honoris Causa. In 2017, the Jagiellonian University at Krakow awarded him the 'Plus Ratio Quam Vis Medal'. His research interests are in the field on non-equilibrium statistical physics and its applications to biology and nanoscience. More information: http://www.ffn.ub.es/webmrubi/ 